Original Research Paper

\title{
Study on Carbon Source Screening Technology for Prevention and Control of Banana Fusarium Wilt
}

\author{
${ }^{1,2}$ Jin Li, ${ }^{1}$ Tingting Duan, ${ }^{1}$ Zhong Lin, ${ }^{1}$ Chao Zheng and ${ }^{2}$ Xiaolin Fan \\ ${ }^{l}$ Guangdong Ocean University, Zhanjiang 524088, China \\ ${ }^{2}$ South China Agricultural University, Guangzhou 510642, China
}

Article history

Received: 02-01-2020

Revised: 22-01-2020

Accepted: 04-03-2020

Corresponding Author:

Xiaolin Fan

South China Agricultural

University, Guangzhou 510642 ,

China

Email:xlfan@scau.edu.cn

\begin{abstract}
With the spread of banana Fusarium wilt and the aggravation of the damage, it has become the biggest threat to the banana industry. Many studies have shown that bio-organic fertilizer plays an important role in the prevention and control of banana wilt, but most studies focused on biocontrol bacterium screening, not screening carbon sources that could prevent banana wilt, which lead to organic fertilizer fertilization without better effect. So that, a pot culture experiment (I) with inoculating Fusarium oxysporum f. sp. Cubense (FOC) on bananas was carried out to screen the carbon sources, and d-malic acid (CS2) to prevent and control banana wilt had been screened out by Biolog technology and Principal Component Analysis of microbial community level physiological profiling. To verify application effects of CS2, another banana pot experiment (II) with FOC inoculation had been done. The results showed that: (1) CS2 can effectively improve the activity of soil microorganisms. (2) CS2 can significantly reduce FOC population in soil, and the FOC population treated with CS2 was respectively lower than that treated with control carbon source (CS1) and without carbon source (CK1) $48 \%$ and $59 \%$. (3) The banana wilt infection rate and disease index of CS2 treatment were significantly smaller than those of CS1 and CK1. (4) CS2 can promote the growth and biomass accumulation of bananas in pathogenic soil. In conclusion, the screening technology studied here can be applied to screen out the carbon sources for prevention of banana Fusarium wilt, which has great significance to the improvement of prevention effect of biological organic fertilizers to banana wilt.
\end{abstract}

Keywords: Carbon Sources, Soil Microbes, FOC, Bananas

\section{Introduction}

Banana Fusarium wilt is a fungal disease of banana and a typical soil-borne vascular bundle disease caused by FOC infection (Wu et al., 2013). This kind of pathogen invades the plant mainly from the root or wound of the banana, through the vascular bundle of banana spreads to the upper part of the pseudostem and leaves and causes the xylem tube be blocked, the nutrition cannot be transmitted normally, finally the plant will withers and dies (Huang et al., 2019). Especially the Fusarium oxysporum f. sp. Cubense species-4 (FOC4), which is the most harmful to bananas, has strong pathogenicity and long survival and latent time, it's difficult to control and can infect almost all kinds of bananas (Li et al., 2015). Therefore, Fusarium wilt caused by FOC4 is currently recognized as a devastating banana disease in the world. It is a worldwide scientific problem that has plagued banana production for many years and also the biggest obstacle to the development of the world banana industry.

At present, the control methods of banana Fusarium wilt mainly include breeding disease-resistant varieties of bananas, killing FOC with chemical agents, and the cultivation measures of preventing and controlling FOC such as rotation, and the biological control methods for inhibiting growth of FOC by exogenous or indigenous antagonistic bacteria. Among them, the biological control represented by biological organic fertilizers with antagonistic bacteria played an important role in inhibiting pathogenic bacteria ( $\mathrm{Wu}$ et al., 2009), moreover, the carbon sources in organic fertilizers can 
significantly improve activity, functional diversity and community structure of soil microbes, and inhibit FOC through beneficial indigenous microorganisms and prevent soil-borne diseases (Lee, 2010). Therefore, biological organic fertilizer not only is more and more important for prevention and control of banana Fusarium wilt, but also is environmentally friendly and pollutionfree, so many scholars have conducted relevant studies on it (Huang et al., 2017). However, most of these studies focused on screening biocontrol bacteria that could inhibit FOC and did not screen organic carbon sources that could prevent and control banana Fusarium wilt according to the metabolic characteristics of soil microorganisms. Microorganisms in different soil have different preferences for organic carbon sources (Dwivedi et al., 2013, Xue et al., 2015), which leads to great differences in the ecological effects of organic fertilizers on soil microorganisms. In addition, the exogenous antagonistic bacteria could not adapt to the new soil environment and colonize in it when they entered the field with the organic fertilizers, thus they could not survive and reproduce normally or play the role of inhibiting pathogens (Shen et al., 2017), which affected the biocontrol effect of organic fertilizers. Therefore, the results of most studies on biological organic fertilizers are only have good effects in the indoor environment, but there are some problems in the field environment, such as the exogenous antagonistic bacteria cannot colonize in the new environment or the additional carbon sources cannot be utilized efficiently by beneficial indigenous microorganisms, which directly lead to the unsatisfactory effect of biological control in the field conditions.

In view of the above problems, according to the carbon metabolism characteristics of microbes in soils inoculated with FOC4 and without FOC4, the carbon source that could prevent and control banana Fusarium wilt was selected by Biolog technology and R language and studied the effect and microbial mechanism of this carbon source on preventing and controlling banana Fusarium wilt through the banana pot experiment. This study will lay a foundation for the prevention and control of banana Fusarium wilt by screening carbon sources and is expected to improve the prevention and control effect of organic fertilizer on banana Fusarium wilt in the field, which is of great significance to maintain the sustainable productivity of bananas in the affected area.

\section{Materials and Methods}

\section{Experimental Materials}

The soil used in these experiments was a sandy loam soil from a farm at South China Agricultural University. The $\mathrm{pH}$ of the soil was 5.98. The soil contained $11.09 \mathrm{~g} / \mathrm{kg}$ organic matter (Potassium bichromate titrimetric method-outside heating method), $52.59 \mathrm{mg} / \mathrm{kg}$ available nitrogen (Alkaline hydrolyzation diffusion method), $56.57 \mathrm{mg} / \mathrm{kg}$ available phosphorus (Olsen's method) and 115.40 $\mathrm{mg} / \mathrm{kg}$ available potassium $\left(\mathrm{NH}_{4} \mathrm{OAc}\right.$ extraction-flame photometry method).

Sterile tissue-cultured banana plants (Musa sp., AAA, Giant Cavendish cv. Baxi,) with 6 leaves were used for the experiments.

Spores of Fusarium oxysporum f. sp. cubense (E. F. Smith) Snyder and Hansen (FOC4) was supplied by the Plant Pathology Lab of the College of Natural Resource and Environment of South China Agriculture University. The concentration of the FOC4 spore suspension was $1 \times 10^{7} \mathrm{cfu} / \mathrm{ml}$.

The carbon sources required for experiment II were selected through experiment I, and they were the control carbon source (CS1) and the carbon source for preventing banana wilt (CS2), respectively. Then, we purchased CS1 and CS2 of analytically pure (AR) from Promega Corporation (USA) as test carbon sources.

\section{Study Design}

\section{Experiment I}

This experiment was a single factor completely randomized design, that is, different FOC concentrations. Thus, there were 3 treatments. Specifically, the tested FOC concentrations were $0,10^{3}$ and $10^{6} \mathrm{cfu}$ per gram of soil, termed $\mathrm{C} 0, \mathrm{C} 1$ and $\mathrm{C} 2$ respectively.

The experiment was performed in a greenhouse at South China Agricultural University (latitude $23^{\circ} 09^{\prime} 40^{\prime \prime} \mathrm{N}$, longitude $113^{\circ} 21^{\prime} 28^{\prime \prime} \mathrm{E}$ ) in November 2014 to January 2015 . Each pot was $15 \times 13 \times 12 \mathrm{~cm}$ and contained $500 \mathrm{~g}$ of the soil with one banana. Each banana had at least 6 leaves at the start of the experiment (12 November 2014). On 22 November 2014, the C0, C1 and C2 FOC spore suspension treatments were added to the $500 \mathrm{mg}$ of the soil around each banana pseudostem from a syringe containing $50 \mathrm{ml}$ of $0,1 \times 10^{4}, 1 \times 10^{7} \mathrm{cfu} / \mathrm{ml}$, respectively.

The $50 \mathrm{ml}$ of $0.6 \%$ compound fertilizer $(22 \mathrm{~N}: 8 \mathrm{P}: 15 \mathrm{~K})$ solution were irrigated into each replication every week. One individual plant was considered a single replication in each treatment and each treatment was replicated 12 times. Thus, there were 3 treatments and 36 plants in this study.

\section{Experiment II}

The experiment was performed in a greenhouse at South China Agricultural University (latitude $23^{\circ} 09^{\prime} 40^{\prime \prime}$ $\mathrm{N}$, longitude $113^{\circ} 21^{\prime} 28^{\prime \prime}$ E) in June to October 2015. Each pot was $16 \times 15 \times 15 \mathrm{~cm}$ and contained $800 \mathrm{~g}$ of the soil with one banana. This experiment sets up 4 treatments, CS1 and CS2 were respectively applied in 
potted plants with inoculating FOC and marked as treatment CS1 and CS2, respectively. Third treatment was with FOC inoculation and without the carbon source as control treatment 1 (CK1). Fourth treatment was without FOC inoculation and the carbon source as control treatment 2 (CK2). Each treatment was repeated 15 times, and one banana was planted in each pot for one repeat, a total of 60 pots were randomly arranged.

The bananas were transplanted into pots on June 19,2015 , and each banana in pot was irrigated once a week with a concentration of $0.4 \%$ NPK compound fertilizer (22N:8P:15K) 100ml. CS1 and CS2 aqueous solutions with concentration of $0.01 \%$ were respectively irrigated in pots of $\mathrm{CS} 1$ and $\mathrm{CS} 2$ treatment every 15 20days and CK1 and CK2 without applying carbon source were instead by clean water. The $100 \mathrm{ml} /$ pot carbon source solution was irrigated every time until the end of the experiment, a total of 8 times of solution or water was irrigated.

The FOC spore suspension $\left(10^{7} \mathrm{cfu} / \mathrm{ml}\right)$ cultured in advance was diluted with sterile water on July 4, 2015 until the spore concentration was $5 \times 10^{5} \mathrm{cfu} / \mathrm{ml}$ (FOC inoculation solution) and then use a syringe respectively inhaled $100 \mathrm{ml}$ FOC inoculation solution and directly injected into the soil around banana pseudostem of treatment needed inoculation. For the treatment without inoculation, inject by $100 \mathrm{ml}$ water instead.

\section{Sample Collection}

\section{Soil Sample Collection}

Experiment I. On 14 January 2015, this is, 54 days after inoculated FOC, soil samples were collected from each of the 12 replicates in each treatment. For each treatment, 3 sets of soil samples were created by randomly combining 4 replicates (three soil samples). The soil samples were stored at $4^{\circ} \mathrm{C}$ for conducting microbial Community Level Physiological Profiling (CLPP) by the Biolog EcoPlate method and the principal components analysis of microbial CLPP was conducted by R software for screening carbon sources of prevention and control FOC.

Experiment II. 107 days after inoculated FOC, soil samples were collected from each of the 15 replicates in each treatment. For each treatment, 3 sets of soil samples were created by randomly combining 5 replicates (three soil samples). The soil samples also were stored at $4^{\circ} \mathrm{C}$ for conducting microbial CLPP and counting the FOC population.

\section{Determination for Plant Growth Indexes and Collection Samples}

Observed the plant height and maximum leaf area of bananas for every ten days in experiment II after inoculated FOC. On the 107th day after inoculation FOC, referenced Carlier's et al. (2002) to statistic the incidence and disease index of bananas when the incidence of banana Fusarium wilt was obvious, and harvested bananas and measured their plant biomass, including root dry weight (DW), shoot DW and plant DW.

\section{Plate Count Method}

Applied the Microbiological Plate Count Method to determine the FOC population in soil and prepared with the selective medium of FOC according to reference literature (Sun et al., 1978).

\section{Biolog Eco Plate Method}

The Biolog technique can be used to investigate the metabolic functional diversity of soil microbes reflected by the utilization ratio of carbon sources (Preston-Mafham et al., 2002). The protocols for creating serially diluted spore suspensions of the soil samples and Biolog EcoPlate method were performed in accordance with Classen's methods (Classen et al., 2010).

Each Biolog EcoPlate consisted of 3 identical sections (3 replicates), each section containing 31 micro wells with distinct dissolved organic carbon substrates and one well with no substrate to serve as negative control (blank; Table 1). For analysis, each plate was treated as 1 individual soil samples for the same treatment was used to fill all wells of the plate. Three replicates in one EcoPlate for each soil samples were detected. The optical densities and Average Well Color Development (AWCD) in the inoculated Biolog EcoPlate was measured at culture times $3,24,36,48,72,96,120,144$ and $168 \mathrm{~h}$ and using a ELx808-Biolog absorbance reader (BioTek Instruments, USA) under a light wavelength of $590 \mathrm{~nm}$.

\section{Statistical Analyses}

Readings from individual plate were inputted into Microsoft Excel for compilation and manipulation. Data from each plate were parsed into 3 data lines for the 3 replicates. For each plate, the Optical Density (OD) of the blank well was subtracted from the OD of each of the 31 wells of its replicate to calculate a net substrate (normalized) OD value (Christian and Lind, 2007). When blanking resulted in negative values, these values were set to zeros for data analysis (Kurten and Barkoh, 2016).

The OD and AWCD of the wells in the Biolog EcoPlate are considered positively associated with the utilization of carbon sources, and reflect the activity of soil microbes. The AWCD conforms to a time-dependent asymptotic sigmoidal curve (Qin et al., 2017). For each replicate reading, the AWCD was calculated as the sum of all normalized OD values divided by 31 (Garland, 1997), as follows:

$$
A W C D=\sum(O D i-R) / n,
$$


where, $O D i$ represents the OD value of the substrate in each well, $R$ represents the OD of the control (blank), ODi-R is the corrected absorbance value (normalized) and $n$ is the 31 types of carbon substrates (Kurten and Barkoh, 2016).

The Principal Components Analysis (PCA) of the OD values at $72 \mathrm{~h}$ of cultivation time was performed with ADE-4 software ( $R$ software package) to study the difference of substrate utilization profiles between the inoculation and non-inoculation treatments. A permutation test was used to test the significance of the groupings suggested by the PCA. Values were considered as different when the probability to reject the nil hypothesis was lower than 0.05 (Dai et al., 2004). Duncan's multiple range tests were performed using SAS statistical software.

\section{Results and Discussions}

\section{FOC Prevention and Control with Carbon Sources Screening}

PCA of soil microbial CLPP was performed to screen the carbon sources for prevention and control FOC. PCA was conducted to understand better the microbial CLPP in the soil microenvironment in response to the different treatments (Hackett and Griffiths, 1997), and microbial utilizing profiles of 31 carbon substrates from different inoculation treatments to analyze associations among these carbon sources and screen the carbon sources (Fig. 1 and Table 1).

To study the effect of $\mathrm{C} 0, \mathrm{C} 1$ and $\mathrm{C} 2$ treatments on soil microbial utilizing profiles to the substrates, a PCA was performed (Fig. 1a and 1b). The results showed that the first principal component (Fig. 1, axis X[PC1]) was mainly and positively determined by the utilization rates of D-Malic acid (30), $\beta$-Methyl-D-glucoside (1) and DCellobiose (24) etc and mainly negatively determined by the utilization rates of 4-Hydroxy benzoic acid (14), its relative inertia was $59.20 \%$. PC2's relative inertia was $10.85 \%$ (Fig. 1, axis $\mathrm{Y}[\mathrm{PC} 2]$ ) and the total relative inertia of $\mathrm{PC} 1$ and $\mathrm{PC} 2$ was $70.05 \%$.

The permutation test of the PCA showed that the differences in the substrate utilization profiles among $\mathrm{C} 0, \mathrm{C} 1$ and $\mathrm{C} 2$ were significant $(P<0.0001)$. The $\mathrm{C} 0$ treatment was clearly distinguished from $\mathrm{C} 1$ and $\mathrm{C} 2$ by their respective substrate utilization profiles, with different utilization rates (Fig. 1b): C0 had higher utilization rates for most carbon sources than did $\mathrm{C} 1$ and C2, especially for D-Malic acid, $\beta$-Methyl-D-glucoside and D-Cellobiose and their distribution of contribution scores on PC1 were 9274, 9237 and 8907, respectively (Table 1). By contrast, utilization rate for 4-Hydroxy benzoic acid of $\mathrm{C} 0$ treatment was considerably lower than those of $\mathrm{C} 2$ and $\mathrm{C} 3$, and distribution of contribution scores from 4-Hydroxy benzoic acid on PC1 was negative $31(-31)$. Therefore, $\mathrm{C} 0$ in the positive half of Axis X could be significantly differentiated from $\mathrm{C} 1$ and $\mathrm{C} 2$ both in the negative half of AxisX, and D-Malic acid (the highest score, 9274) was selected as the carbon source of FOC prevention and control (CS2), 4-Hydroxy benzoic acid (the lowest score, -31) was selected as the carbon source of control (CS1).
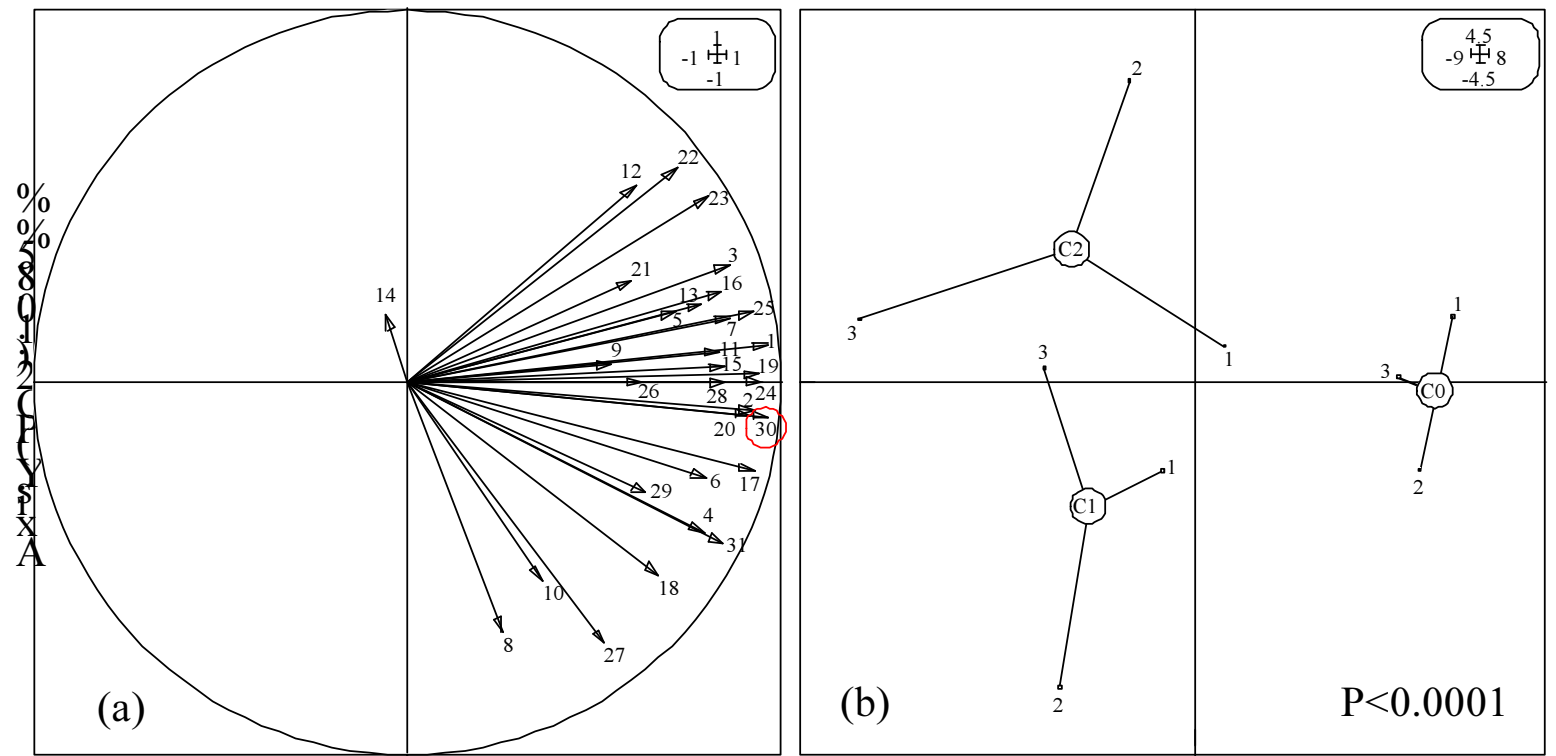

AxisX(PC1):59.20\%

Fig. 1: PCA of soil microbial CLPP in C0, C1 and C2 treatments. (a) Loading plot of 31 substrates by PCA. (b) PCA scores plot of microbial substrate utilization in different treatments. Note: The key for the identification numbers of the 31 substrates are listed in Table 1 . The encircled carbon substrate in red circle was selected as the tested carbon source (CS2) 
Table 1: Relative contributions of the carbon substrates on PC1

\begin{tabular}{|c|c|c|c|c|c|}
\hline \multicolumn{3}{|c|}{96 EcoPlate cells } & \multirow{2}{*}{$\begin{array}{l}\text { Substrate } \\
\text { None (water blank) }\end{array}$} & \multirow{2}{*}{$\begin{array}{l}\text { Substrate ID } \\
\text { None }\end{array}$} & \multirow{2}{*}{$\begin{array}{l}\text { Contribution score } \\
\text { None }\end{array}$} \\
\hline A1 & A5 & A9 & & & \\
\hline $\mathrm{A} 2$ & A6 & A10 & $\beta$-Methyl-D-glucoside & 1 & 9237 \\
\hline $\mathrm{A} 3$ & A7 & A11 & D-Galactonic acid $\gamma$-Lactone & 2 & 8566 \\
\hline A4 & A8 & A12 & L-Arginine & 3 & 7365 \\
\hline B1 & B5 & B9 & Pyruvic acid methyl ester & 4 & 6270 \\
\hline B2 & B6 & $\mathrm{B} 10$ & D-Xylose & 5 & 5145 \\
\hline B3 & B7 & $\mathrm{B} 11$ & D-Galacturonic acid & 6 & 6365 \\
\hline B4 & B8 & $\mathrm{B} 12$ & L-Asparagine & 7 & 7421 \\
\hline $\mathrm{C} 1$ & $\mathrm{C} 5$ & C9 & Tween 40 & 8 & 649 \\
\hline $\mathrm{C} 2$ & C6 & $\mathrm{C} 10$ & I-Erythritol & 9 & 2981 \\
\hline $\mathrm{C} 3$ & $\mathrm{C} 7$ & $\mathrm{C} 11$ & 2-Hydroxy benzoic acid & 10 & 1278 \\
\hline $\mathrm{C} 4$ & $\mathrm{C} 8$ & $\mathrm{C} 12$ & L-Phenylalanine & 11 & 6908 \\
\hline D1 & D5 & D9 & Tween 80 & 12 & 3688 \\
\hline $\mathrm{D} 2$ & D6 & D10 & D-Mannitol & 13 & 6143 \\
\hline D3 & D7 & D11 & 4-Hydroxy benzoic acid & 14 & $-31^{b}$ \\
\hline D4 & D8 & D12 & L-Serine & 15 & 7149 \\
\hline E1 & E5 & E9 & $\alpha$-Cyclodextrin & 16 & 7053 \\
\hline E2 & E6 & E10 & N-Acetyl-D-glucosamine & 17 & 8602 \\
\hline E3 & E7 & E11 & $\gamma$-Hydroxybutyric acid & 18 & 4495 \\
\hline E4 & E8 & E12 & L-Threonine & 19 & 8787 \\
\hline F1 & F5 & F9 & Glycogen & 20 & 8368 \\
\hline $\mathrm{F} 2$ & F6 & F10 & D-Glucosaminic acid & 21 & 3553 \\
\hline F3 & F7 & F11 & Itaconic acid & 22 & 5171 \\
\hline $\mathrm{F} 4$ & F8 & $\mathrm{F} 12$ & Glycyl-L-glutamic acid & 23 & 6481 \\
\hline G1 & G5 & G9 & D-Cellobiose & 24 & 8907 \\
\hline $\mathrm{G} 2$ & G6 & G10 & Glucose-1-phosphate & 25 & 8543 \\
\hline G3 & G7 & G11 & $\alpha$-Ketobutyric acid & 26 & 3902 \\
\hline G4 & G8 & G12 & Phenylethylamine & 27 & 2777 \\
\hline $\mathrm{H} 1$ & H5 & H9 & $\alpha$-D-Lactose & 28 & 7205 \\
\hline $\mathrm{H} 2$ & H6 & $\mathrm{H} 10$ & D, L- $\alpha$-Glycerol phosphate & 29 & 4080 \\
\hline $\mathrm{H} 3$ & $\mathrm{H} 7$ & H11 & D-Malic acid & 30 & $9274^{a}$ \\
\hline $\mathrm{H} 4$ & $\mathrm{H} 8$ & $\mathrm{H} 12$ & Putrescine & 31 & 7098 \\
\hline
\end{tabular}

Note: Each Biolog EcoPlate contains 96 wells consisting of three replicates (first 3 columns) of 31 substrates and 1 water blank. ${ }^{\mathbf{a}}$ is the carbon substrate that contributed the most on PC1, ${ }^{\mathbf{b}}$ is the carbon substrate that contributed the least on PC1.

\section{Effects of the Carbon Sources on the Plant Height and Maximum Leaf Area}

Plant height and leaf area of banana were important indicators of banana growth. The trend of plant height and maximum leaf area of banana with the extension of inoculation days was shown in Fig. 2a and 2b. The plant height of each treatment increased with the growth time and the plant height of CS2 treatment was significantly higher than that of CS1, CK2 and CK1 treatments during the whole culture period (Fig. 2a). The bananas treated by CS2 were respectively higher than CS1, CK2 and CK1 $3 \%, 5 \%$ and $11 \%$ at the end of the experiment (107th day after inoculation).

The maximum leaf area of each treatment increased rapidly with the growth time for 57 days after inoculation (Fig. 2b). However, the maximum leaf area of the inoculated banana (CS1, CS2, CK1) grew slowly or stopped after 57days of inoculation, but the leaf area of the non-inoculated banana (CK2) still increased rapidly. Because the inoculated bananas did not get sick or the disease was relatively mild in first 57days and the growth of banana leaves was not seriously affected. However, the banana began to get sick and the disease seriously affected the growth of banana leaves after 57days. From 57th day to the end of the experiment (107th day), the area of banana leaves without inoculated (CK2) was significantly larger than that inoculated, while that inoculated with CS2 was significantly larger than that inoculated with CS1 and CK1. In conclusion, the growth of bananas treated with carbon source CS2 and those not inoculated (CK2) were better than those treated with other treatments, indicating that the treatment with carbon source CS2 was beneficial to the growth of bananas in the diseased soil and the effect of promoting the growth of bananas was better than that with carbon source CS1.

\section{Effects of the Carbon Sources on Banana Biomass}

As shown in Table 2, there was no significant difference in banana root DW among treatments, however, shoot DW and plant DW of bananas of CS2 and CK2 were significantly larger than those of CS1 and $\mathrm{CK} 1$, the plant DW of CS2 was respectively larger than 
CS1 and CK1 $16.82 \%$ and $15.90 \%$. These results indicated that carbon source CS2 could promote banana growth and improve biomass in the disease soil, but carbon source CS1 could not. This may be because the application of carbon source CS2 can effectively reduce the incidence and harm of bananas wilt and contribute to the healthy growth of bananas.

\section{Effects of the Carbon Sources on Wilt Infection and Disease Index of Fusarium wilt in Banana Plants}

Wilt Infection and disease index of bananas can directly reflect the disease incidence of bananas (Carlier et al., 2002). As shown in Fig. 3, the bananas of non-inoculated treatment (CK2) did not infect disease at the end of the pot experiment, therefore wilt infection and disease index was significantly smaller than other inoculation treatments. Wilt infection of bananas treated with CS2 was significantly lower than those of CS1 and CK1 during inoculation treatment, which respectively lowers than CS1 and CK1 40 and 46 percentage points. The disease index of bananas treated with CS2 was also significantly lower than that of CK1, lower than that of CK1 $70 \%$, and the difference between CS1 and CK1 was not significant. As a result, the bananas treated with CS2 were less infectious than CS1 and CK1, this indicates that the application of CS2 can reduce the occurrence of banana Fusarium wilt, which may be because the CS2 improved the microbial structure by regulating the metabolic activity of soil microorganisms and contributed to beneficial microorganisms to inhibit the growth of FOC, thus decreased FOC population and reducing the incidence of bananas Fusarium wilt (Wu et al., 2009).

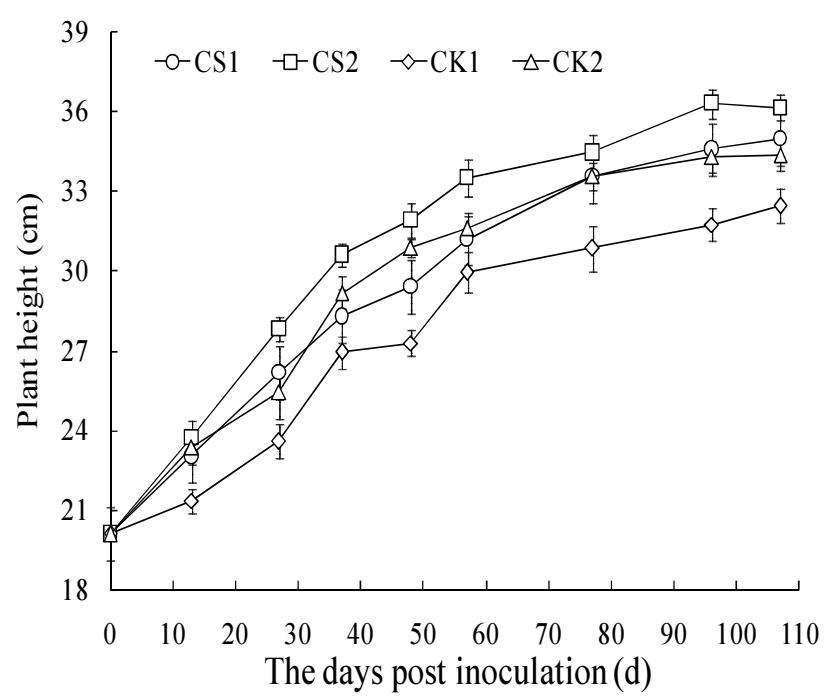

(a)



(b)

Fig. 2: (a) Effects of carbon sources on the plant height; (b) Effects of carbon sources on the maximum leaf area 


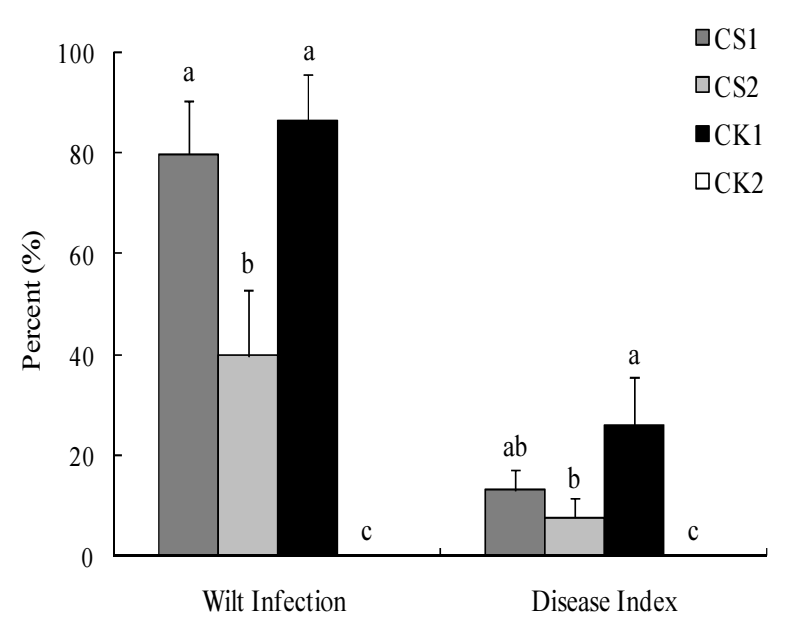

Fig. 3: Effects of carbon sources on wilt infection and disease index of Fusarium wilt

Table 2: Effects of the carbon sources on banana dry weight $(\mathrm{g})$

\begin{tabular}{llll}
\hline Treatment & Shoot DW & Root DW & Plant DW \\
\hline CK1 & $18.65 \pm 1.16 \mathrm{~b}$ & $1.68 \pm 0.10 \mathrm{a}$ & $20.32 \pm 1.21 \mathrm{~b}$ \\
CS1 & $18.75 \pm 0.54 \mathrm{~b}$ & $1.41 \pm 0.11 \mathrm{a}$ & $20.16 \pm 0.61 \mathrm{~b}$ \\
CS2 & $21.94 \pm 0.46 \mathrm{a}$ & $1.61 \pm 0.11 \mathrm{a}$ & $23.55 \pm 0.48 \mathrm{a}$ \\
CK2 & $21.24 \pm 0.52 \mathrm{a}$ & $1.55 \pm 0.09 \mathrm{a}$ & $22.80 \pm 0.52 \mathrm{a}$ \\
\hline
\end{tabular}

Note: Number in the table is mean \pm standard deviation. Different letters in each column meant significant differences among treatments $(P<0.05)$.

\section{Effects of the Carbon Sources on the FOC Population in Soil}

The FOC population is directly related to the incidence of banana Fusarium wilt (Sun et al., 1978). In general, the more FOC population in soil, the more serious the incidence of banana disease, therefore, it is of great significance to analyze the FOC population in different treatments soil for the study of carbon sources prevention and control of banana Fusarium wilt. As shown in Fig. 4, FOC population in soil without inoculated (CK2) was significantly smaller than that in other inoculated. In the inoculation treatment, the FOC population in the soil treated with CS2 was significantly lower than that treated without carbon source (CK1), and respectively less than that treated with CK1 and CS1 $59 \%$ and $48 \%$, while the difference between CS1 and CK1 was not significant. This result verified that carbon source CS2 could prevent and control the occurrence of banana Fusarium wilt by reducing the FOC population in soil, because CS2 could provide preferred carbon source nutrients for soil microorganisms, improved their overall activity and diversity, and thus inhibited the growth of FOC (Lee, 2010).

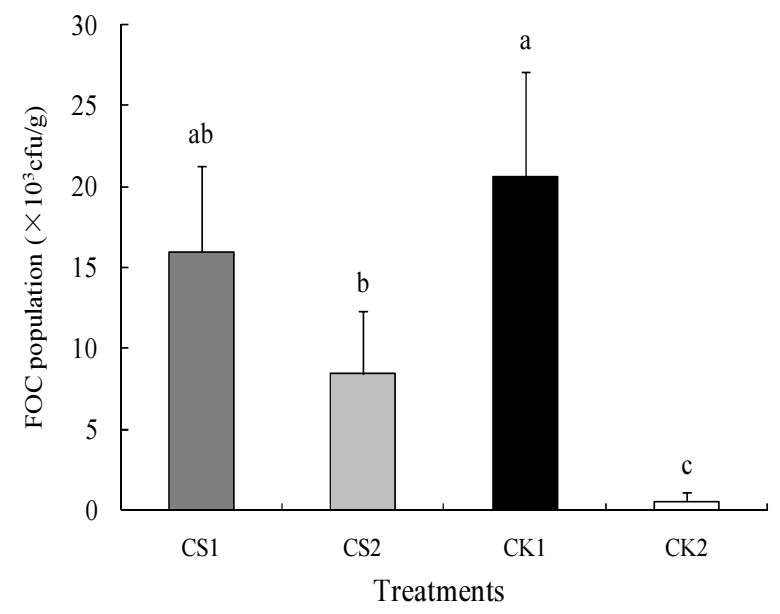

Fig. 4: Effects of carbon sources on the FOC population

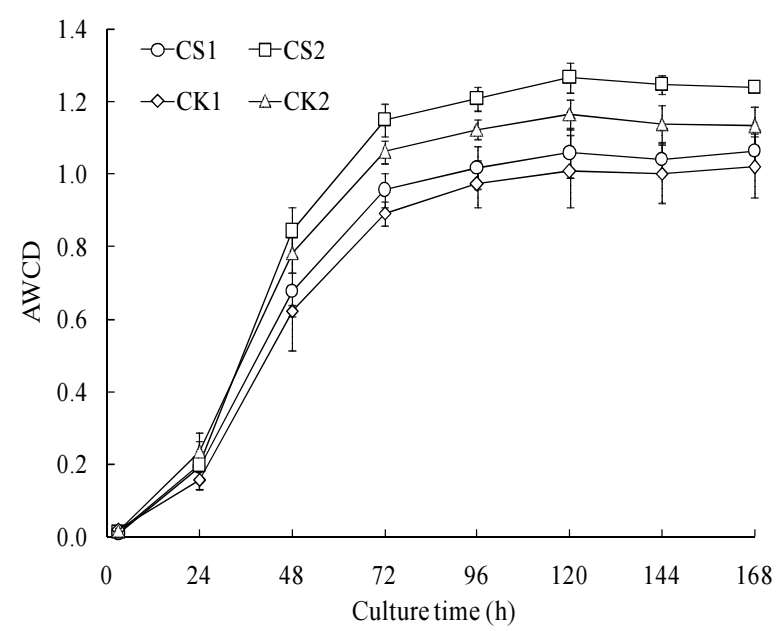

Fig. 5: AWCD variation of total carbon substrates with cultivation time prolonged

\section{Effects of the Carbon Sources on AWCD Variation of Total Carbon Substrates}

The AWCD can reflect the carbon utilization profile and activity of soil microbes (Qin et al., 2017). In general, the AWCD in this study increased in "S" curve during cultivation (Fig. 5). The AWCD curves of 4 treatments showed that carbon utilization increased slowly in the first $24 \mathrm{~h}$, rapidly from 24 to $72 \mathrm{~h}$ and was fairly stable from 72 to $168 \mathrm{~h}$. An analysis according to the different treatments showed that carbon utilization in soil treated with CS2 was significantly higher than that of CS1, CK1 and CK2 in interval of $48 \mathrm{~h}$ to $168 \mathrm{~h}$, and the carbon utilization in CK2 soil was significantly higher than that of CS1 and CK1 soils. This result means that the activity of soil microbes is lowered by the presence of FOC (CK1), but is improved with the use of carbon source CS2. This result may be due to the 
FOC is the main dominant population under the inoculation condition and it will inevitably compete with other microorganisms for the habitats such as carbon sources, thus resulting in insufficient nutrition and space for the growth of other microorganisms. It is also possible that the large amount of FOC produces a substance that inhibits the growth of other microorganisms (Huang et al., 2015). And applying $\mathrm{CS} 2$ could provide carbon nutrient preferred by most microorganisms in the soil for the growth of microorganisms (Fig. 1), thus improved the overall activity of soil microbes (Lee, 2010).

\section{Conclusion}

In this paper, studied carbon source screening technology for the prevention and control of banana Fusarium wilt, and screened carbon source CS2 to control of banana wilt according to this technology. This carbon source could effectively improve the overall activity of microorganisms in pathogenic soil, and the AWCD treated with CS2 was significantly higher than that treated with other treatments. Meanwhile, CS2 could significantly reduce the FOC population in soil, the FOC population treated with CS2 was respectively lower than that treated with control carbon source (CS1) and without carbon source (CK1) 48\% and 59\%. Therefore, the banana wilt infection of CS2 was significantly smaller than that of $\mathrm{CS} 1$ and $\mathrm{CK} 1$, which respectively lower than CS1 and CK1 40 and 46 percentage points. The disease index of bananas wilt treated by CS2 was also significantly lower than that of CK1, which lower than that of CK $170 \%$, and the difference between CS1 and CK1 was not significant. Why could CS2 reduce the FOC population, wilt infection rate and disease index of bananas? Those details were not covered in this paper and will be covered in a future study.

Due to apply CS2 could effectively prevent and control the occurrence of banana Fusarium wilt and reduce the damage of Fusarium wilt to bananas, the CS2 could promote the growth and biomass accumulation of bananas in the pathogenic soil. The results showed that the growth vigor of bananas treated with CS2 was significantly better than that of CS1 and CK1 and the shoot DW and plant DW of bananas were significantly larger than those of CS1 and CK1. The plant DW of CS2 was respectively larger than that of CS1 and CK1 $16.82 \%$ and $15.90 \%$. In conclusion, this paper studied the carbon source screening technology according to microbial CLPP in different soil, and this screening technology can be used to screen out high-quality carbon sources for the prevention and control of banana wilt, which has great significance to the production or selection of organic fertilizers containing high-quality carbon sources and the improvement of prevention effect of biological organic fertilizers to banana wilt.

\section{Acknowledgement}

This work was supported by National Natural Science Fund of China (NSFC31071857, NSFC 30871594), China Agricultural Research System (nycytx-33-07), China's Ministry of Agriculture Special Fund for Agro-scientific Research in Public Interest (A13174), Innovating and Strengthening Colleges Project of Guangdong (2018KQNCX104), Scientific Research Foundation for Ph.D of Guangdong Ocean University (E15038).

\section{Author's Contributions}

Jin Li and Tingting Duan: Designed and performed the experiments, analyzed the data and prepared the paper. Both authors contributed equally to this work.

Zhong Lin and Chao Zheng: Participated to collect the materials related to the experiment.

Xiaolin Fan: Designed the experiments and revised the manuscript.

\section{Ethics}

The authors declare their responsibility for any ethical issues that may arise after the publication of this manuscript.

\section{Conflict of Interest}

The authors declare that they have no competing interests. The corresponding author affirms that all of the authors have read and approved the manuscript.

\section{References}

Carlier, J., D.D. Waele and J. Escalant, 2002. Global evaluation of Musa germplasm for resistance to Fusarium wilt, Mycosphaerella leaf spot diseases and nematodes. Bioversity Int., 2002: 67-67.

Christian, B.W. and O.T. Lind, 2007. Multiple carbon substrate utilization by bacteria at the sedimentwater interface: Seasonal patterns in a stratified eutrophic reservoir. Hydrobiologia, 586: 43-56. DOI: $10.1007 / \mathrm{s} 10750-006-0476-6$

Classen, A.T., S.I. Boyle, K.E. Haskins, S.T. Overby and S.C. Hart, 2010. Community-level physiological profiles of bacteria and fungi: plate type and incubation temperature influences on contrasting soils. Fems Microbiol. Ecol., 44: 319-328.

DOI: 10.1016/S0168-6496(03)00068-0

Dai, J., T. Becquer, J.H. Rouiller, G. Reversat and F. Bernhard-Reversat et al., 2004. Heavy metal accumulation by two earthworm species and its relationship to total and DTPA-extractable metals in soils. Soil Biol. Biochem., 36: 91-98.

DOI: 10.1016/j.soilbio.2003.09.001 
Dwivedi, M.C., A.K. Singh and S.S. Singh, 2013. Effect of organic manures and fertilizers on the growth, yield and disease incidence of fusarium wilt in black gram. Progressive Agric., 13: 23-24.

Garland, J.L., 1997. Analysis and interpretation of community-level physiological profiles in microbial ecology. Fems Microbiol. Ecol., 24: 289-300.

DOI: $10.1111 / \mathrm{j} .1574-6941.1997 . t b 00446 . x$

Hackett, C.A. and B.S. Griffiths, 1997. Statistical analysis of the time-course of Biolog substrate utilization. J. Microbiol. Meth., 30: 63-69.

DOI: $10.1016 / \mathrm{S} 0167-7012(97) 00045-6$

Huang, J., Y. Pang, F. Zhang, F. Zhang and Q. Huang et al., 2019. Suppression of Fusarium wilt of banana by combining acid soil ameliorant with biofertilizer made from Bacillus velezensis H-6. Eur. J. Plant Pathol., 154: 585-596.

DOI: $10.1007 / \mathrm{s} 10658-019-01683-5$

Huang, N., W. Wang, Y. Yao, F. Zhu and W. Wang et al., 2017. The influence of different concentrations of bio-organic fertilizer on cucumber Fusarium wilt and soil microflora alterations. Plos One, 12: e171490-e171490.

DOI: 10.1371/journal.pone.0171490

Huang, X.Q., L.L. Liu, T. Wen, R. Zhu and J. Zhang et al., 2015. Illumina MiSeq investigations on the changes of microbial community in the Fusarium oxysporum f.sp. cubense infected soil during and after reductive soil disinfestation. Microbiol. Res., 181: 33-42.

DOI: $10.1016 /$ j.micres.2015.08.004

Kurten, G.L. and A. Barkoh, 2016. Evaluation of community-level physiological profiling for monitoring microbial communityfunction in aquaculture ponds. North Am. J. Aquaculture, 78: 34-44. DOI: 10.1080/15222055.2015.1079580

Lee, J., 2010. Effect of application methods of organic fertilizer on growth, soil chemical properties and microbial densities in organic bulb onion production. Scientia Horticulturae, 124: 299-305. DOI: $10.1016 /$ j.scienta.2010.01.004

Li, W.M., M. Dita, W. Wu, G.B. Hu and J.H. Xie et al., 2015. Resistance sources to Fusarium oxysporum $\mathrm{f}$. sp. cubense tropical race 4 in banana wild relatives. Plant Pathol., 64: 1061-1067. DOI: $10.1111 /$ ppa. 12340

Preston-Mafham, J., L. Boddy and P.F. Randerson, 2002. Analysis of microbial community functional diversity using sole-carbon-source utilisation profiles - a critique. Fems Microbiol. Ecol., 42: 1-14.

DOI: 10.1111/j.1574-6941.2002.tb00990.x
Qin, X.M., Y. Zheng, L. Tang and G.Q. Long, 2017. Crop rhizospheric microbial community structure and functional diversity as affected by maize and potato intercropping. J. Plant Nutrit., 40: 2402-2412. DOI: 10.1080/01904167.2017.1346674

Shen, Z.Z., L. Sun, D.S. Wang, N.N. Lyu and C. Xue et al., 2017. Effects of lime-ammonium bicarbonate fumigation and biofertilizer application on Fusarium wilt and biomass of continuous cropping cucumber and watermelon. Chinese J. Applied Ecol., 28: 3351-3359. DOI: $10.13287 /$ j.1001-9332.201710.036

Sun, E.J., H.J. Su and W.H. Ko, 1978. Identification of Fusarium oxysporum f. sp. cubense race 4 from soil or host tissue by cultural characters. Phytopathology, 68: 1672-1673.

DOI: 10.1094/Phyto-68-1672

Wu, H.S., J.Q. Yang, W.G. Miao, W.G. Miao and N. Ling et al., 2009. Suppression of Fusarium wilt of watermelon by a bio-organic fertilizer containing combinations of antagonistic microorganisms. BioControl, 54: 287-287. DOI: $10.1007 / \mathrm{s} 10526-008-9168-7$

Wu, Y., G. Yi, X. Peng, B. Huang and E. Liu et al., 2013. Systemic acquired resistance in Cavendish banana induced by infection with an incompatible strain of Fusarium oxysporum f. sp. cubense. J. Plant Physiol., 170: 1039-1046.

DOI: 10.1016/j.jplph.2013.02.011

Xue, C., C. Ryan Penton, Z.Z. Shen, R. Zhang and Q. Huang et al., 2015. Manipulating the banana rhizosphere microbiome for biological control of Panama disease. Scientific Rep., 5: 11124-11124. 